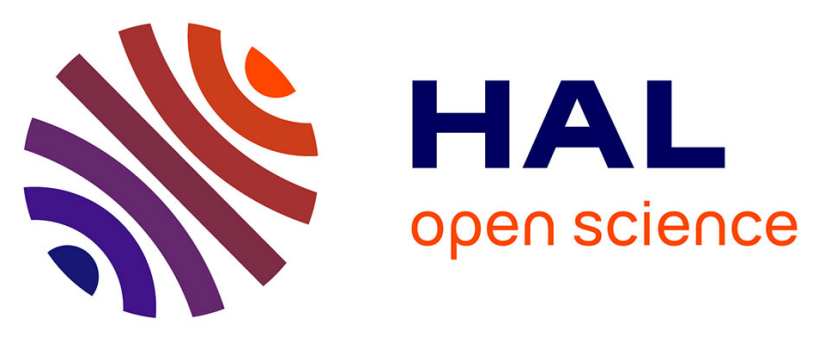

\title{
Hydrophilicity-Hydrophobicity Transformation, Thermoresponsive Morphomechanics, and Crack Multifurcation Revealed by AIEgens in Mechanically Strong Hydrogels
}

\author{
Yubing B Hu, Lucile Barbier, Zhao Li, Xiaofan F Ji, Heiva Le Blay, \\ Dominique Hourdet, Nicolas Sanson, Jacky W y Lam, Alba Marcellan, Ben \\ Zhong Tang
}

\section{- To cite this version:}

Yubing B Hu, Lucile Barbier, Zhao Li, Xiaofan F Ji, Heiva Le Blay, et al.. HydrophilicityHydrophobicity Transformation, Thermoresponsive Morphomechanics, and Crack Multifurcation Revealed by AIEgens in Mechanically Strong Hydrogels. Advanced Materials, 2021, pp.2101500. 10.1002/adma.202101500 . hal-03357267

\section{HAL Id: hal-03357267}

https://hal-espci.archives-ouvertes.fr/hal-03357267

Submitted on 30 Sep 2021

HAL is a multi-disciplinary open access archive for the deposit and dissemination of scientific research documents, whether they are published or not. The documents may come from teaching and research institutions in France or abroad, or from public or private research centers.
L'archive ouverte pluridisciplinaire HAL, est destinée au dépôt et à la diffusion de documents scientifiques de niveau recherche, publiés ou non, émanant des établissements d'enseignement et de recherche français ou étrangers, des laboratoires publics ou privés. 
DOI: $10.1002 /(($ please add manuscript number $))$

Article type: Full Paper

Hydrophilicity-Hydrophobicity Transformation, Thermoresponsive Morphomechanics and Crack Multifurcation Revealed by AIEgens in Mechanically Strong Hydrogels

Yubing Hu, Lucile Barbier, Zhao Li, Xiaofan Ji, Heiva Le Blay, Dominique Hourdet, Nicolas Sanson, Jacky W. Y. Lam*, Alba Marcellan*, and Ben Zhong Tang*

Dr. Y. B. Hu, Dr. Z. Li, Dr. X. F. Ji, Dr. J. W. Y. Lam, Prof. B. Z. Tang

Department of Chemistry, the Hong Kong Branch of Chinese National Engineering Research Center for Tissue Restoration and Reconstruction, Institute for Advanced Study, The Hong Kong University of Science and Technology, Guangdong-Hong Kong-Macro Joint Laboratory of Optoelectronic and Magnetic Functional Materials, Clear Water Bay, Kowloon, Hong Kong 999077, China

E-mail: chjacky@ust.hk (J. W. Y. Lam), tangbenz@ust.hk (B. Z. Tang)

L. Barbier, H. L. Blay, D. Hourdet, N. Sanson, A. Marcellan

ESPCI ParisTech, PSL Research University, Sorbonne Universités, UPMC Univ. Paris 06, Laboratoire Sciences et Ingénierie de la Matière Molle CNRS UMR CNRS 7615, 10 Rue Vauquelin F-75231, Paris

E-mail: alba.marcellan@espci.fr(A. Marcellan)

Dr. Y. B. Hu, Dr. J. W. Y. Lam, Prof. B. Z. Tang

HKUST-Shenzhen Research Institute, No. 9 Yuexing 1st RD, South Area, Hi-tech Park, Nanshan, Shenzhen 518057, China

Prof. B. Z. Tang

Center for Aggregation-Induced Emission, from Molecular Aggregates, SCUT-HKUST Joint Research Institute, State Key Laboratory of Luminescent Materials and Devices, South China University of Technology, Guangzhou 510640, China

Prof. B. Z. Tang

AIE institute, Guangzhou Development Distinct, Huangpu, Guangzhou 510530, China 


\begin{abstract}
Biomimetic exploration of stimuli-responsive and crack-resistant hydrogels is of great academic and practical significance, although the rational design of tough hydrogels is limited by insufficient mechanism study due to the lack of imaging techniques to "see" hydrogels at mesoscale level. We designed a series of composite hydrogels with compartmentalized thermal response by incorporating aggregation- and polarity-sensitive fluorescent probes in a poly $(N$-isopropylacrylamide) (PNIPAM) network grafted with poly $(N, N$-dimethylacrylamide) side-chains. We explore the fluorescence technique as a powerful tool to directly visualize their hydrophilicity-hydrophobicity transformation and the composition-dependent microphase separation. Based on the morphological observation and mechanical measurements, the concept of morphomechanics with a comprehensive mechanism clarification is proposed. In this regard, the thermoresponsive toughening are attributed to the formation of multiple noncovalent interactions and the conformational changes of PNIPAM chains. The enhanced fracture energy by crack multifurcation is related to the tearing-like disruption of weak interfaces between the separated phases.
\end{abstract}

Keywords: hydrophilicity-hydrophobicity transformation, microphase separation, crack multifurcation, aggregation-induced emission 


\section{Introduction}

Wet and soft matter is ubiquitous in nature. In living matter, structure and function are intimately linked to the environment. Many recent technologies are based on hydrogel responsiveness and imply hydrogel integration into biomedical devices ${ }^{[1]}$, soft actuators ${ }^{[2]}$ or bioelectronics $^{[3]}$ that require demanding mechanical behavior. Mechanical weaknesses of synthetic hydrogels, in terms of fracture stiffness, toughness, adhesion or excessive swelling, are often addressed as limitation factor to practical development. In contrast, living matter has optimized the tissues with diverse hierarchical compartmentalized structure and adjustable mechanical properties to realize their functionalities in a changing environment. ${ }^{[4]}$ Many forced-related biological tissues, such as spider silk, muscle and tendon, are assembled from a variety of ordered protein fibers in aqueous media and exhibit intricate adaptive swelling and mechanical behaviors under stimuli. Moreover, these fibrillar soft tissues develop extremely high fracture toughness (given their high water content) and demonstrate an intriguing damage behavior by tearing along the interfaces between fibers under excessive tension to prevent injury to the entire tissue (Figure 1a). ${ }^{[5-8]}$ From a structural perspective, dynamic noncovalent interactions and conformational variations of proteins play a crucial role in their strong and tunable mechanical properties. ${ }^{[9-10]}$ Although a deep understanding of the relationship between their molecular structure, supramolecular interactions and mechanical behavior remain a big challenge, it is still fascinating for synthetic scientists and engineers to develop bioinspired high-strength and stimuli-responsive hydrogels. ${ }^{[1]}$

To meet the ever-increasing requirements, continuous effort has been devoted to the development of high-strength hydrogels. ${ }^{[12-13]}$ Among them, the design original polymer network topologies, as double-networks ${ }^{[14]}$, sliding-ring networks ${ }^{[15]}$, tetra-PEG networks ${ }^{[16]}$, or the introduction of structural and dynamical heterogeneities, as in nanocomposite gels ${ }^{[17-18]}$, have shown significant mechanically enhancement than their conventional counterparts. 
Despite of these great achievements, biomimetic approach can offer a broad platform to explore energy dissipation pathways for crack-resistant hydrogels. ${ }^{[19-20]}$ Recently, proteininspired phase-separated hydrogels have been proved to enhance the toughness of polymer networks ${ }^{[21-22]}$. A systematic crack bifurcation has been also reported to prevent crack fracture of the whole phase-separated hydrogels, which is promising to mimic the tearing behavior in hydrogels (Figure 1b). ${ }^{[23]}$ To describe such behavior of sacrificing the noncovalent interactions or weak interfaces to preserve the integrity of the whole, morphomechanics is proposed here to draw on the mechanical breakage of intramolecular bonds in mechanochemistry. ${ }^{[24]}$ While the network topology and fiber-like formation of phase-separated domains, appears to be a key-aspect on fracture toughness enhancement, ${ }^{[25-26]}$ the observation of the phase separation morphology and the understanding of the strengthening mechanism are still insufficient. Moreover, the current understanding of toughening scenarios is often deduced from the chemical nature of network structure and the macroscopic properties. ${ }^{[27-29]}$ So far, the coupling effects due to noncovalent interactions and morphological structures on the mechanical property are still under debate due to the lack of mesoscale imaging techniques. It appears essential to develop effective techniques to "see" the mesoscale morphologies in various hydrated state and to clarify their roles in fracture toughening. ${ }^{[30-31]}$

In this framework, many modern microscopic techniques including scanning electron microscopy, transmission electron microscopy and atomic force microscopy are inadequate. These cost and time-consuming imaging techniques are generally performed in a vacuum state and have destructive sample preparation processes. On the contrary, fluorescence technique offers a direct approach to visualize the analytes in a simple, sensitive, highcontrast and onsite manner. However, many traditional fluorophores exhibit weak or no emission in the solid state and aqueous environment due to the aggregation-caused quenching effect. ${ }^{[32]}$ Fortunately, this negative effect can be overcome by luminogens (AIEgens) with aggregation-induced emission (AIE), which are generally non-emissive in dilute solutions but 
emit intensely in the aggregated state due to the restriction of intramolecular motions. ${ }^{[33]}$ AIEgens are often embedded with electron-donating (D) and accepting (A) units to trigger polarity-responsive emission change assisted by the twisted intramolecular charge transfer (TICT) effect. ${ }^{[34]}$ Herein, we developed a new polymerizable fluorescent probe with AIE and TICT characters for the direct visualization of microphase separation patterns and hydrophilicity-hydrophobicity transformation in thermoresponsive composite hydrogels. Combined with their mechanical behaviors, thermoresponsive toughening and crack multibifurcation, we gain a deep understanding of their structure-property relationships and propose a detailed strengthening mechanism of hydrogels.

\section{Results and Discussions}

\subsection{Fluorescent Probe with Response to Aggregation and Polarity Change}

To visualize the morphological change of stimuli-responsive hydrogels, we designed and synthesized a new water-soluble AIEgen named TVPA with the chemical structure shown in Figure 2a. The electron-donating triphenylamine and the electron-withdrawing pyridinium unit are connected to the ethylene core, which endows TVPA with molecular rotors, a D-A structure and ionic character. The three-step synthetic route are provided in Figure S1. The reaction intermediates and TVPA were fully characterized by NMR and mass spectroscopies (Figure S2-9). The theoretical frontier molecular orbital distribution was calculated by density functional theory calculations [DFT, m062x/6-311G (d, p)]. As shown in Figure 2a, the highest occupied molecular orbital (HOMO) mainly localizes in the triphenylamine unit and the lowest unoccupied molecular orbital (LUMO) mainly locates in the pyridinium unit. Such electron separation of HOMO and LUMO indicates a $\pi-\pi^{*}$ transition with a charge transfer character.

The photoluminescence (PL) and the quantum yields (QYs) in water, glycerol and the solid state of TVPA was first investigated in to verify the desired optical properties. As shown in Figure $2 b$, the water-soluble TVPA exhibits weak red emission in dilute aqueous solution 
but emits intensely in viscous glycerol and in the solid state with 87 and 54 times higher intensity, respectively. The corresponding QYs of TVPA are also increased from $0.4 \%$ to $10.7 \%$ and $6.1 \%$, respectively, confirming the AIE characteristic of TVPA because of the restriction of intramolecular motions. Considering the D-A structure of TVPA, we recorded its PL spectra and fluorescent photos in different solvents and polymer films with various polarities. Figure 2c demonstrates the remarkable solvatochromic effect of TVPA: its emission color gradually shifts from blue $\left(\lambda_{\mathrm{em}}=436 \mathrm{~nm}\right)$ to red $\left(\lambda_{\mathrm{em}}=610 \mathrm{~nm}\right)$ when the solvent is changed from non-polar toluene to polar methanol. The TVPA-doped polymer films also display the same bathochromic shift from blue emission $\left(\lambda_{\mathrm{em}}=457 \mathrm{~nm}\right)$ in nonpolar polybutadiene to red emission $\left(\lambda_{\mathrm{em}}=621 \mathrm{~nm}\right)$ in polar poly(ethylene glycol) (PEG) (Figure 2d). Such polarity-responsive behaviors clearly manifest the TICT characteristic of TVPA. In short, we successfully synthesized a AIEgen with desired strong emission in the aggregated state and high sensitivity to environmental polarity. From synthetic prospective, acrylate-containing TVPA probe is promising to be covalently incorporated in polymer network by olefinic polymerization in aqueous media. Such characteristics make it a promising fluorescent sensor to the crowdedness and polarity change in macromolecular systems.

\subsection{Fluorescent response in stimuli-responsive hydrogels}

Based on the previous efforts in phase-separated hydrogels, we attempted to incorporate TVPA in the chemically crosslinked poly( $N$-isopropylacrylamide) (PNIPAM) network grafted with poly $(N, N$-dimethylacrylamide) (PDMA) side-chains as schematically illustrated in Figure 3a. The PDMA macromonomer was synthesized according to the procedure reported in Figure S10. Gels synthesis was carried out in water. 2-mm thick hydrogel plates were prepared by free radical copolymerization of NIPAM, TVPA, PDMA macromonomer and $N, N^{\prime}$-methylenebisacrylamide (MBA) as chemical crosslinker using a redox initiation at room temperature with potassium peroxodisulfate (KPS) and $N, N, N^{\prime}, N^{\prime}$-tetramethylethylenediamine 
(TEMED). We anticipated that the copolymer network can alternate from the as-prepared homogeneous state at room temperature to a biphasic state under thermal stimulus above the lower critical solution temperature of PNIPAM (LCST, $T_{\mathrm{c}} \approx 32{ }^{\circ} \mathrm{C}$ ) and back to the homogeneous state below the LCST. ${ }^{[35]}$ Indeed, the well-hydrated PNIPAM chains should undergo a coil-to-globule transition and collapse into PNIPAM-rich hydrophobic domains above the LCST. At the same time, the water released is absorbed by the hydrophilic PDMA phase which thus makes it possible to preserve the swelling of the gel (as polymer concentration) at macroscopic scale by osmotic contribution. Thus, from preparation state, gels can undergo microphase separation at almost constant macroscopic volume for $\mathrm{T}>\mathrm{T}_{\mathrm{c}}$. Meanwhile, the covalently incorporated TVPA is expected to exhibit fluorescence in terms of both intensity and color changes, from weakly red-emissive in the extended hydrophilic PNIPAM chains to intensively blue-emissive in the crowded hydrophobic PNIPAM-rich domains. Using this design principle, three hydrogels (GN2D3, GN3D3 and GN6D3) were achieved from NIPAM (N) network and grafted PDMA macromonomers (D) with different weight ratios (N/D: 2/3, 3/3 and 6/3) while keeping the same PNIPAm network (content and crosslink density) (Figure S11 and Table S1). For comparison purposes, GN and GD hydrogels were prepared from NIPAM monomers and DMA monomers with TVPA, respectively.

The macroscopic properties of three hydrogels were investigated at different temperatures by swelling experiments, transmittance and PL measurements. Starting from the as-prepared (reference) state, as expected, the three hydrogels largely swell when equilibrated in water at $20{ }^{\circ} \mathrm{C}$ and the level of swelling at equilibrium increases with the PDMA content (Figure S12). In comparison, the swelling of hydrogels equilibrated at $60{ }^{\circ} \mathrm{C}$ remains closer to the reference state, either above, similar or below for GN2D3, GN3D3 and GN6D3, respectively. Such swelling behavior implies that they successfully inherit the thermoresponsive properties from LCST-type PNIPAM and their swelling ability is tunable by changing the weight fraction of 
PNIPAM and PDMA. From the absorption spectra, the change in transmittance at $600 \mathrm{~nm}$ was determined as a function of temperature between $20{ }^{\circ} \mathrm{C}$ and $60{ }^{\circ} \mathrm{C}$ (Figure S13). For all the samples, GN2D3, GN3D3 and GN6D3, the transmittance drops dramatically between 24 and $34{ }^{\circ} \mathrm{C}$ with a visible change from transparent to opaque. This macroscopic observation, common to the three hydrogels, clearly indicates the transition from homogeneous to heterogeneous system due the formation of PNIPAM-rich domains above their LCST. ${ }^{[36]}$

As the incorporation of TVPA into the hydrogels provides direct access to the fluorescence technique in hydrogels, we carefully studied the PL of the three hydrogels in the preparation state and after heating in water for $30 \mathrm{~min}$. As indicated in Figure 3b, all the PL spectra of GN2D3, GN3D3 and GN6D3 display remarkable both intensity and color changes at $T_{\mathrm{c}}$. By heating from $20^{\circ} \mathrm{C}$ to $60{ }^{\circ} \mathrm{C}$, the PL intensity increases by up to 17 times and the PL maximum shifts from $620 \mathrm{~nm}$ to $480 \mathrm{~nm}$. The reversible fluorescence change between dark orange $\left(20^{\circ} \mathrm{C}\right)$ and bright blue $\left(60^{\circ} \mathrm{C}\right)$ is observable by naked eyes. Among the three hydrogels, GN6D3 with the highest composition of PNIPAM shows strongest intensity enhancement owing to its slight macroscopic shrinkage. GN2D3 has the largest blue shift to $470 \mathrm{~nm}$, which indicates that PNIPAM chains have collapsed into strongly dehydrated and dense domains. To better understand the source of fluorescence change, we studied the PL of GN and GD at $20^{\circ} \mathrm{C}$ and $60{ }^{\circ} \mathrm{C}$. As shown in Figure $3 \mathrm{c}$ and $3 \mathrm{~d}$, the temperature only exerts a strong influence on the fluorescence of GN. The emission color of GN at the temperature above $T_{\mathrm{c}}$ is close to that of traditional non-polar polymers. Referring to the AIE and TICT characteristics of TVPA probe, such enhanced and blue-shifted fluorescence underlines the coil-to-globule conformational change and implies the transformation from hydrophilic PNIPAM chains into hydrophobic PNIPAM domains above the LCST. In other words, TVPA can serve as a simple and effective reporter to micro-environmental changes in hydrogels, especially to the transformation from molecular hydrophilicity to morphological hydrophobicity. 
To demonstrate this newly proposed concept, additional proof-of-concept experiments were performed on stimuli-responsive gelation of TVPA-containing synthetic polymers, synthetic-natural complex and natural polymers. In Figure 3e, the thermo-responsive gelation of poly(ethylene glycol)-block-poly(propylene glycol)-block-poly(ethylene glycol) (PEGPPG-PEG) solution displays a gradual blue-shifted emission when its aqueous solution is heated, traducing dehydration level. A mixture of weakly red-emissive $\alpha$-cyclodextrin $(\alpha-C D)$ and PEG solutions turns into a hydrogel after the formation of an inclusion complex $\alpha-C D-$ PEG, which exhibits a strong orange emission (Figure 3f). Again, with the diffusion of sodium hydroxide, the chitosan solution gradually gels with the shift of the emission color from red to orange (Figure 3g). Collectively, these emission behaviors show that TVPA can sensitively detect both environmental concentration and polarity changes, respectively by intensity and color-shift emission, taking place in stimuli-responsive hydrogels. More generally, the TVPA probe allows for probing the hydrophilic-hydrophobic transformation in biological macromolecular systems and would make it possible to assess the transition level through the polarity of the environment as a function of the emission color.

\subsection{Fluorescent imaging of microphase separated hydrogels}

Based on the preliminary results, the fluorescence microscope was employed to systematically investigate the thermoresponsive morphology of the hydrogels at the mesoscopic scale (Figure S14). The GN3D3 hydrogel, selected as representative sample for the analysis of thermal effect, was studied a) in the preparation state, b) after immersing in water at $60{ }^{\circ} \mathrm{C}$ for $30 \mathrm{~min}$ and c) after overnight recovery at $20{ }^{\circ} \mathrm{C}$, respectively. Under thermal cycling, fluorescent images of GN3D3 switch reversibly between chromatic-orange and chromatic-blue emission, which is consistent with the macroscopic observation. The appearance of Newton's rings with multicolor fringes illustrates the existence of light interference. By applying simple optics principles ${ }^{[37]}$, we can draw two guidelines: 1) the numbers of interference fringes in Newton's rings is positively correlated with the actual size 
of the corresponding objects; 2) the distance between two constructive fringes of same color, called as fringe spacing, depends on the refractive index of local polymer domains. The role of light interference in the optical observation of the fluorescent composite hydrogels is interpretated in detail (Figure S15). The interference patterns in Figure 4a display continuous irregular combinations of Newton's rings, which is associated with some spatial heterogeneities distribution of partially hydrated polymer phase and aqueous phase trapped during polymerization. ${ }^{[38]}$ Noted that a morphological pattern with two distinct regions in Figure $4 \mathrm{~b}$ is generated by heating GN3D3 above the LCST while its volume change at the macroscopic level is almost negligible. Region I displays plenty of strongly emissive spherical domains, which result from the large difference in refractive index between polymer concentrated domains (PNIPAM-rich) and water (or highly hydrated polymer regions). Region II is a more uniform pattern displaying weakly emissive matrix corresponding to the domains where the highly hydrated PDMA domains exhibit a refractive index comparable to water. Such assignment of region I and region II are supported by further comparison with the morphologies of GD and GN after immersing in water at $60{ }^{\circ} \mathrm{C}$ (Figure S16). After the thermal cycling, an entire Newton's ring spreads over the entire area in the recovered pattern with relatively wide fringe spacing, suggesting a more homogenous network produced by rehydration of PNIPAM domains and rearrangement of both PDMA and PNIPAM chains (Figure 4c). By immersing GN3D3 in water at different temperature, the scale of phase transition and the size of PNIPAM-rich domains are continuously increasing from 25 to $65{ }^{\circ} \mathrm{C}$ (Figure S16). This series of interference patterns obtained by thermal cycling demonstrates the reliability of fluorescent observations and the reversibility of the system.

The fluorescence images of GN2D3, GN3D3 and GN6D3 were then taken to compare the impact of gel topology over the microphase separation patterns. As presented in Figure $4 \mathrm{~d}-\mathrm{i}$, the ratio of PNIPAM network and PDMA grafts exerts a great influence on the phaseseparated morphologies. In GN2D3 with PDMA as the major component, a uniform 
dispersion of the PDMA-rich matrix with isolated PNIPAM-rich spherical domains is observed (Figure 4d and 4g). From GN2D3 to GN3D3, the content of hydrophilic grafts is decreased, and the PNIPAM-rich spherical domains appear to be more interconnected (Figure 4b, 4e and 4h). The picture for GN6D3 (Figure 4f and 4i) appears more complex with even larger polymer-rich domains interconnected. From the morphological changes, we deduce that the PNIPAM network collapses through the pearl necklace-like formation of intrachain micelles and the spacer between micelles becomes closer as the content of hydrophilic PDMA grafts decreases. In fact, these phase-separated patterns provide good experimental evidence for the theoretical models of sparsely grafted amphiphilic copolymer self-assemblies. ${ }^{[39]}$ In terms of Newton's rings assigned to PNIAM-rich domains, the numbers of interference fringe and the fringe spacing gradually increase from GN2D3, GN3D3 to GN6D3. This suggests that the PNIPAM-core micelles become larger and more uniform with increasing composition of PNIPAM. These observations demonstrate that the AIE-assisted fluorescent imaging offers a simple, high-contrast and onsite access to visualize stimuli-responsive morphological variations and differentiate the composition-dependent phase-separated patterns. In this way, fluorescence technique affords a new way of revealing mesostructures in hydrogels which had never been observed so far.

\subsection{Mechanical response and fracture mode of fiber-like gels}

After exploring the stimuli-responsive and composition-dependent microphase separation of GN2D3, GN3D3 and GN6D3, we investigate the impact of such morphologies on the mechanical behavior. The temperature-induced viscoelastic properties were first studied under small deformations, probing the mechanical response of gel's microstructure without strong perturbations from its conformation at rest. Note that samples were tested under isolated conditions, i.e. immersed in paraffin oil, to prevent water evaporation or swelling during experiment. The frequency-dependence of their viscoelastic behaviors at $20{ }^{\circ} \mathrm{C}$ and $60{ }^{\circ} \mathrm{C}$ was examined to verify their temperature-dependent viscoelastic moduli (Figure S17). Then the 
thermo-responsive mechanical properties at small deformations were measured by temperature sweep at a constant frequency $(f=1 \mathrm{rad} / \mathrm{s})$. As illustrated in Figure $5 \mathrm{a}$, heating the hydrogel samples from $20{ }^{\circ} \mathrm{C}$ to $60{ }^{\circ} \mathrm{C}$ triggers a dramatic increase of the elastic modulus (G') corresponding to the emergence and progress of microphase separation, where the elastic PNIPAM network collapses into a stiffened phase. The rheological measurement under heating and cooling process shows excellent superposition performance, implying the recovery and stability of the system at a slow rate of temperature change $\left(0.25^{\circ} \mathrm{C} / \mathrm{min}\right)$. As expected from optical observation of phase-separated patterns, the addition of PDMA grafts implies a limitation of phase separation domains - from virtually continuous to more isolated domains - and results in a decreased reinforcement efficiency, in terms of $G^{\prime}$ for $T>T_{\text {c }}$. Sufficiently far from $T_{\mathrm{c}}$, the contribution of dissipative contribution remains quite low, the loss modulus $\left(G^{\prime \prime}\right)$ being one order of magnitude lower than G'.

We then investigated the large strain behaviors of GN2D3, GN3D3 and GN6D3 at $20{ }^{\circ} \mathrm{C}$ and $60{ }^{\circ} \mathrm{C}$ by tensile stress-strain measurements (Figure 5b-5d and Figure S18-S19). As shown in Figure $5 \mathrm{~b}$, at $20^{\circ} \mathrm{C}$, all the samples show the typical response of lightly covalently crosslinked polymer networks. Indeed, the PNIPAM network below $T_{\mathrm{c}}$ is supposed to feature entropy-driven elasticity. At $20^{\circ} \mathrm{C}$, Young's modulus $(E)$ is rather similar, independently of network topologies, and the obtained values are in line with viscoelastic data $\left(E \cong 3 G^{\prime}\right)$. Conversely, at $60{ }^{\circ} \mathrm{C}$ stress-strain curves significantly overestimate the purely entropic contribution of the network at $20{ }^{\circ} \mathrm{C}$. Such remarkable changes on fracture strain and stress are ascribed to the conformational entanglement of the collapsed PNIPAM chains and the formation of multiple noncovalent interactions in PNIPAM domains above LCST, respectively. GN6D3 displays the highest Young's modulus of $18.3 \mathrm{kPa}$ at $60{ }^{\circ} \mathrm{C}$, which is consistent with the role of PNIPAM as a thick continuous matrix as observed at mesoscale. In addition, an interesting crack deflection occurs when stretching GN6D3 to large strain (Figure S19). To graphically depict their divergence from entropy-driven elasticity, stress-strain 
behaviors have been plotted as reduced stress versus reciprocal elongation $(1 / \lambda)$ according to semi-empirical model. ${ }^{[40]}$ As shown in Figure 5d, the almost horizontal lines at $20{ }^{\circ} \mathrm{C}$ indicate that their original mechanical performance is dominated by entropic elasticity. In contrast, at $60{ }^{\circ} \mathrm{C}$ their reduced stress $(1 / \lambda)$ curves obviously deviate from entropic elasticity, displaying roughly softening and hardening behaviors. The softening behavior is contributed from the disruption of noncovalent interactions that dissipate energy during the unravelling of the collapsed PNIPAM network from globule conformation to stretched state. After the consumption of physical associations, the polymer chains are oriented and aligned along the stretching direction and account for the final hardening behavior before fracture.

Tensile tests on notched specimen were performed at $60{ }^{\circ} \mathrm{C}$ to focus on the crack propagation and fracture toughening mechanisms. The notch was made with a razor blade to initiate the crack propagation where the notch induces high strain amplification. The tensile stress-strain curves are plotted in Figure 5e and the corresponding stretching processes are recorded using pictures (Figure 5g-i) and videos (Videos S1-3). All GNxDy topologies exhibit strong resistance to crack propagation by shielding the crack tip singularity: first by crack blunting, followed by systematic crack furcation perpendicularly to the plane of the notch. Interestingly, pre-notched specimens in Figure 5e sustain higher fracture stresses than their un-notched counterparts in Figure 5b, suggesting that toughening mechanisms are operating more efficiently under severe loading, as knotty-tearing fracture mode in natural rubbers. ${ }^{[41]}$ As depicted in Figure 5g, GN2D3 with pre-crack displays blunting and one crack deflection along the stretching direction. GN3D3 displays three consecutive crack deflections and GN6D3 is further developed into crack multifurcation, i.e. five crack branches are noticed (Figure $5 \mathrm{~h}$ and $5 \mathrm{i}$ ). This multifurcation behavior exactly mimics the tearing behavior of fibrillar tissues and inherits the advantage of keeping the integrity of the material as much as possible. In laminate materials, theoretical criterion for crack deflection is reached when the adhesive energy of the interface becomes less than the cohesive energy in a certain ratio. ${ }^{[42]}$ In 
our case, as the composition of PNIPAM increases from GN2D3 to GN6D3, the cohesive energy of hydrogels is intensified and the opportunity of encountering the PNIPAM/PDMA interfaces along the crack path also raises. This explains the increasing process of crack multifurcation observed from GN2D3, GN3D3 to GN6D3. The fracture energies of notched specimens, which describes the strain energy required to propagate the crack across hydrogels, have been estimated in Figure 5f. With crack multifurcation, GN6D3 displays the highest fracture energy at around $800 \mathrm{~J} / \mathrm{m}^{2}$. This is a quite high value considering the high hydration level of the gel, as the fracture energy for unfilled rubber is around $1000 \mathrm{~J} / \mathrm{m}^{2}$. The key-point, here, relies on multiple fracture surface area that are generated during the failure process as additional energy dissipation pathway which resembles the fiber-assembled biological tissues. Acquiring such ability to resist crack penetration and maintain the integrity of the entire body by partial sacrifice in strengthened hydrogels are of great significance for material engineering and practical applications.

\subsection{Mechanism study}

Based on the above studies, going from microscopic hydrophilicity-hydrophobicity transformation to mesoscopic morphological variation and up to macroscopic mechanical performance, we propose a toughening mechanism as illustrated in Figure 6. So far, it has been widely accepted that the LCST behavior of PNIPAM is associated with the "hydrophobic interactions" of the hydrophobic backbone and isopropyl groups ${ }^{[43-45]}$, but this is a limited view. According to the microenvironmental polarity change indicated by TVPA probe, the PNIPAM network has completely transformed from hydrophilicity to hydrophobicity under thermal stimulus (Figure 6a). By analyzing the molecular structure of PNIPAM, the amide groups present favorable interactions with water molecules through hydrogen bonds at low temperature and promote water solubility of PNIPAM chains. However, above the LCST, the coil-to-globule transition generates the collapsed PNIPAM domains with extensive intra- and inter-chain hydrogen bonds between amide groups, which 
will occupy the previous hydrogen-bond acceptors or donors for water and make the whole PNIPAM domains water insoluble. The dehydration of $\mathrm{C}=\mathrm{O}$ and $\mathrm{N}-\mathrm{H}$ groups and the formation of new hydrogen bonds between amide groups are further proved by the temperature-dependent infrared spectroscopy measurements (Figure S20). In this way, the PNIPAM domains are strengthened significantly due to the massive generation of noncovalent interactions, mainly including hydrogen bonds. Of course topological constraints implied by the distributions of network mesh-sizes and hydrophilic grafts make the morphology of the phase separation more complex.

Under uniaxial mechanical stretching, the strengthened phase-separated hydrogels gradually break noncovalent interactions and dissipate the strain energy during the unravelling of the collapsed PNIPAM domains (Figure 6b). The orientation and alignment of polymer network with the reversible formation of new noncovalent interactions in highly stretched hydrogels, generate fiber-like structures and lead to an efficient resistance to crack propagation. Hence, the mechanical performance of the stimuli-responsive phase-separated hydrogels is improved just by heating when the macroscopic volume change is balanced by the swelling of PDMA domains. The role of fiber-like microphase separation is well demonstrated in tensile mode on pre-notched specimens. As shown in Figure 6c, the crack propagation along the horizontal direction is blocked when the crack tip meets a weak interface between PNIPAM and PDMA phases. Since the adhesive energy of PNIPAM/PDMA interfaces is incomparable to the cohesive energy, the crack deflects along the vertical stretching direction. Meanwhile, the concentrated tensile stress activates the chain orientation and alignment around the initial crack tip where strain amplification takes place. In contrast, the weaker areas at the edges of the hydrogel are cracked into additional notches, probably in the PDMA-rich swollen phase. In other words, the heterogeneous distribution of dense polymer-rich domains and weak highly hydrated phases promotes the formation of tearing-like crack multifurcation phenomenon, which efficiently enlarges the mechanical 
strain energy required to propagate the crack across the sample. Furthermore, the composition-dependence of the crack multifurcation is related to the thickness and the interface to volume ratio of fibrillar structures, which is predictable from their microphase separation patterns. Briefly, the above-mentioned mechanical behaviors of sacrificing the noncovalent interactions or weak interfaces between two separated phases in order to retard the fracture failure and preserve the integrity of the whole exactly match the proposed concept of morphomechanics.

\section{Conclusion}

In summary, we have successfully realized the direct visualization at mesoscale level of microphase separation with easy differentiation of hydrophobic collapsed PNIPAM domains in gels by developing a fluorescent indicator with both AIE and TICT characters that can be easily integrated into the PNIPAM network by polymerization in aqueous medium. The presence of hydrophilic macromonomer grafts in the network prevents the macroscopic collapse of the hydrogel and helps to the generation of hierarchical fibrillary structures. The microphase separation can be readily identified at the mesoscale as spherical domains with different interconnected modes in a facile, high-contrast and noninvasive manner. These observations under fluorescence microscopy revealed dense and hydrophobic mesostructures which had never been observed so far.

The overall mechanism study has been well summarized by the proposed concept of morphomechanics. In contrast to the general understanding of "hydrophobic interactions", the transformation from molecular hydrophilicity to morphological hydrophobicity has been attributed to the formation of multiple non-covalent interactions. The universality of these transformations by several stimuli-responsive sol-gel transitions, such concept could meet interests on materials science and biology communities. The crack multifurcation has been associated the fibrillary structures by direct observation of microphase separation patterns, which provides an additional energy dissipation pathway and enhances fracture energy. The 
mesoscopic exploration of stimuli-responsive materials remains a key-point to the design of high-strength gels or more biomimetic materials. For instance, for a better understanding of how biopolymers regulate their inter and intra assemblies via phase transition to perform various biological functions such as reaction acceleration, signal transduction and transcriptional regulation. ${ }^{[46]}$

\section{Supporting Information}

The Supporting Information is available free of charge.

\section{Conflict of Interest}

The authors declare no conflict of interest.

\section{Acknowledgments}

We are grateful for the financial support from the National Science Foundation of China (21788102, 21490570, and 21490574), the Research Grant Council of Hong Kong (16305618, 16304819, N-HKUST609/19, and C6009-17G), the Science and Technology Plan of Shenzhen (JCYJ20160229205601482, JCYJ20170818113602462, JCYJ20180306180231853, and JCYJ 20180306174910791), the Innovation and Technology Commission (ITC-CNERC14SC01), and National Key Research and Development Program of China (2018YFE0190200), and ESPCI Paris - Sorbonne Université - CNRS. Y.B.H. also thanks Mohamed Hanafi, Guylaine Ducouret, Xiaolin Liu, Junkai Liu, Louis Debertrand, Zuxiang Xu and Qingyun Li for their technical assistance.

\section{Author Contributions}

B.Z.T. and A.M. initiated the international collaboration to study the inner structure and mechanical mechanism of hydrogels. Y.B.H. proposed this specific idea, performed all experiments and wrote the manuscript. B.Z.T. and A.M. provided their kind guidance and financial support. A.M., J.W.Y.L., D.H. and N.S. offered many suggestions for the revisions 
of manuscript. L.B., Z.L., X.F.J. and H.L.B. teach the experimental details to Y.B.H. from synthesis to mechanical measurement.

Received: ((will be filled in by the editorial staff))

Revised: ((will be filled in by the editorial staff)) Published online: ((will be filled in by the editorial staff))

\section{References}

[1] S. I. Park, D. S. Brenner, G. Shin, C. D. Morgan, B. A. Copits, H. U. Chung, M. Y. Pullen, K. N. Noh, S. Davidson, S. J. Oh, Nat. Biotechnol. 2015, 33, 1280.

[2] M. Wehner, R. L. Truby, D. J. Fitzgerald, B. Mosadegh, G. M. Whitesides, J. A. Lewis, R. J. Wood, Nature 2016, 536, 451.

[3] H. Yuk, B. Lu, X. Zhao, Chem. Soc. Rev. 2019, 48, 1642.

[4] W. Huang, D. Restrepo, J. Y. Jung, F. Y. Su, Z. Liu, R. O. Ritchie, J. McKittrick, P. Zavattieri, D. Kisailus, Adv. Mater. 2019, 31, 1901561.

[5] C. Riekel, C. Craig, M. Burghammer, M. Müller, Naturwissenschaften 2001, 88, 67.

[6] J. E. Muscolino, Kinesiology-E-Book: The Skeletal System and Muscle Function. Elsevier Health Sciences: 2014.

[7] R. Bahr, S. Mæhlum, Clinical Guide to Sports Injuries. Human Kinetics: 2004.

[8] P. K. Hansma, G. E. Fantner, J. H. Kindt, P. J. Thurner, G. Schitter, P. J. Turner, S. F. Udwin, M. M. Finch, J Musculoskelet Neuronal Interact. 2005, 5, 313.

[9] T. Scheibel, Curr. Opin. Biotechnol. 2005, 16, 427.

[10] M. J. Buehler, S. Keten, Nano Res. 2008, 1, 63.

[11] U. G. Wegst, H. Bai, E. Saiz, A. P. Tomsia, R. O. Ritchie, Nat. Mater. 2015, 14, 23.

[12] C. W. Peak, J. J. Wilker, G. Schmidt, Colloid. Polym. Sci. 2013, 291, 2031.

[13] X. Zhao, Soft Matter 2014, 10, 672.

[14] J. P. Gong, Y. Katsuyama, T. Kurokawa, Y. Osada, Adv. Mater. 2003, 15, 1155. 
[15] Y. Okumura, K. Ito, Adv. Mater. 2001, 13, 485.

[16] T. Sakai, T. Matsunaga, Y. Yamamoto, C. Ito, R. Yoshida, S. Suzuki, N. Sasaki, M. Shibayama, U.-i. Chung, Macromolecules 2008, 41, 5379.

[17] S. v. Rose, A. Dizeux, T. Narita, D. Hourdet, A. Marcellan, Macromolecules 2013, 46, 4095.

[18] K. Haraguchi, T. Takehisa, Adv. Mater. 2002, 14, 1120.

[19] J. Kopeček, Biomaterials 2007, 28, 5185.

[20] J. S. Mohammed, W. L. Murphy, Adv. Mater. 2009, 21, 2361.

[21] T. Nonoyama, Y. W. Lee, K. Ota, K. Fujioka, W. Hong, J. P. Gong, Adv. Mater. 2020, $32,1905878$.

[22] J. L. Holloway, A. M. Lowman, G. R. Palmese, Soft Matter 2013, 9, 826.

[23] H. Guo, N. Sanson, D. Hourdet, A. Marcellan, Adv. Mater. 2016, 28, 5857.

[24] S. L. James, C. J. Adams, C. Bolm, D. Braga, P. Collier, T. Friščić, F. Grepioni, K. D. Harris, G. Hyett, W. Jones, Chem. Soc. Rev. 2012, 41, 413.

[25] C. Mussault, H. Guo, N. Sanson, D. Hourdet, A. Marcellan, Soft Matter 2019, 15, 8653.

[26] H. Guo, C. Mussault, A. Brûlet, A. Marcellan, D. Hourdet, N. Sanson, Macromolecules 2016, 49, 4295 .

[27] Q. Chen, H. Chen, L. Zhu, J. Zheng, J. Mater. Chem. B 2015, 3, 3654.

[28] B. Wu, W. Chassé, R. Peters, T. Brooijmans, A. A. Dias, A. Heise, C. J. Duxbury, A. P. Kentgens, D. F. Brougham, V. M. Litvinov, Macromolecules 2016, 49, 6531.

[29] E. Ducrot, Y. Chen, M. Bulters, R. P. Sijbesma, C. Creton, Science 2014, 344, 186.

[30] K. Li, Y. Lin, C. Lu, Chem. Asian J. 2019, 14, 715.

[31] Z. Wang, J. Nie, W. Qin, Q. Hu, B. Z. Tang, Nat. Commun. 2016, 7, 1.

[32] T. Förster, K. Kasper, Z. Elektrochem, Ber. Bunsenges. Phys. Chem. 1955, 59, 976.

[33] J. Mei, N. L. Leung, R. T. Kwok, J. W. Lam, B. Z. Tang, Chem. Rev. 2015, 115, 11718. 
[34] R. Hu, E. Lager, A. Aguilar-Aguilar, J. Liu, J. W. Lam, H. H. Sung, I. D. Williams, Y. Zhong, K. S. Wong, E. Pena-Cabrera, J. Phys. Chem. C 2009, 113, 15845.

[35] M. Heskins, J. E. Guillet, J. Macromol. Sci. A 1968, 2, 1441.

[36] S. Nayak, S. B. Debord, L. A. Lyon, Langmuir 2003, 19, 7374.

[37] M. Born, E. Wolf, Principles of optics: electromagnetic theory of propagation, interference and diffraction of light. Elsevier: 2013.

[38] M. Shibayama, Macromolecular Chemistry Physics 1998, 199, 1.

[39] O. Borisov, E. Zhulina, Macromolecules 2005, 38, 2506.

[40] M. Mooney, J. Appl. Phys. 1940, 11, 582.

[41] G. R. Hamed, Rubber Chem. Technol. 1994, 67, 529.

[42] K. Kendall, P. Roy. Soc. A-Math. Phy. 1975, 344, 287.

[43] C. Wu, S. Zhou, Macromolecules 1995, 28, 8381.

[44] E. C. Cho, J. Lee, K. Cho, Macromolecules 2003, 36, 9929.

[45] A. Halperin, M. Kröger, F. M. Winnik, Angew. Chem. Int. Ed. 2015, 54, 15342.

[46] E. J. N. Dolgin, Nature 2018, 555, 300. 


\section{Figures}
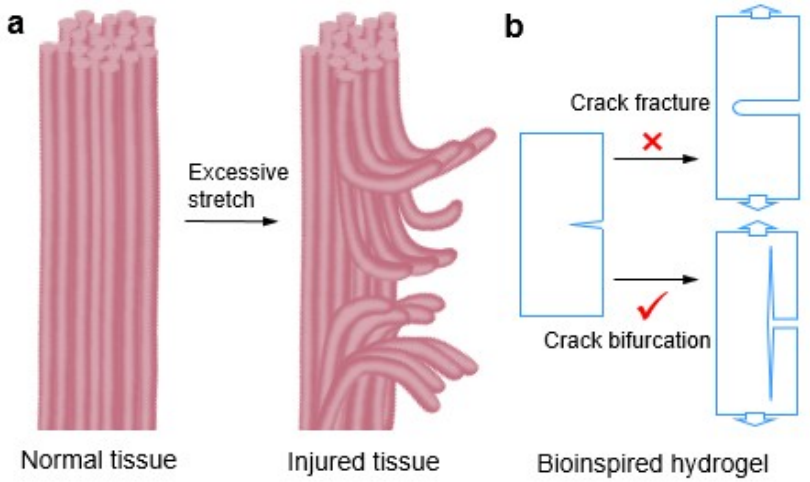

Figure 1. Biomimetic design of high-strength hydrogels. a, Simplified tearing model of fibrillar soft tissues $\frac{5-8}{5-8}$ under excessive tension. $\mathbf{b}$, Bioinspired design for crack-resistant hydrogels by deflecting the fracture along the stretching axis through crack bifurcation. 

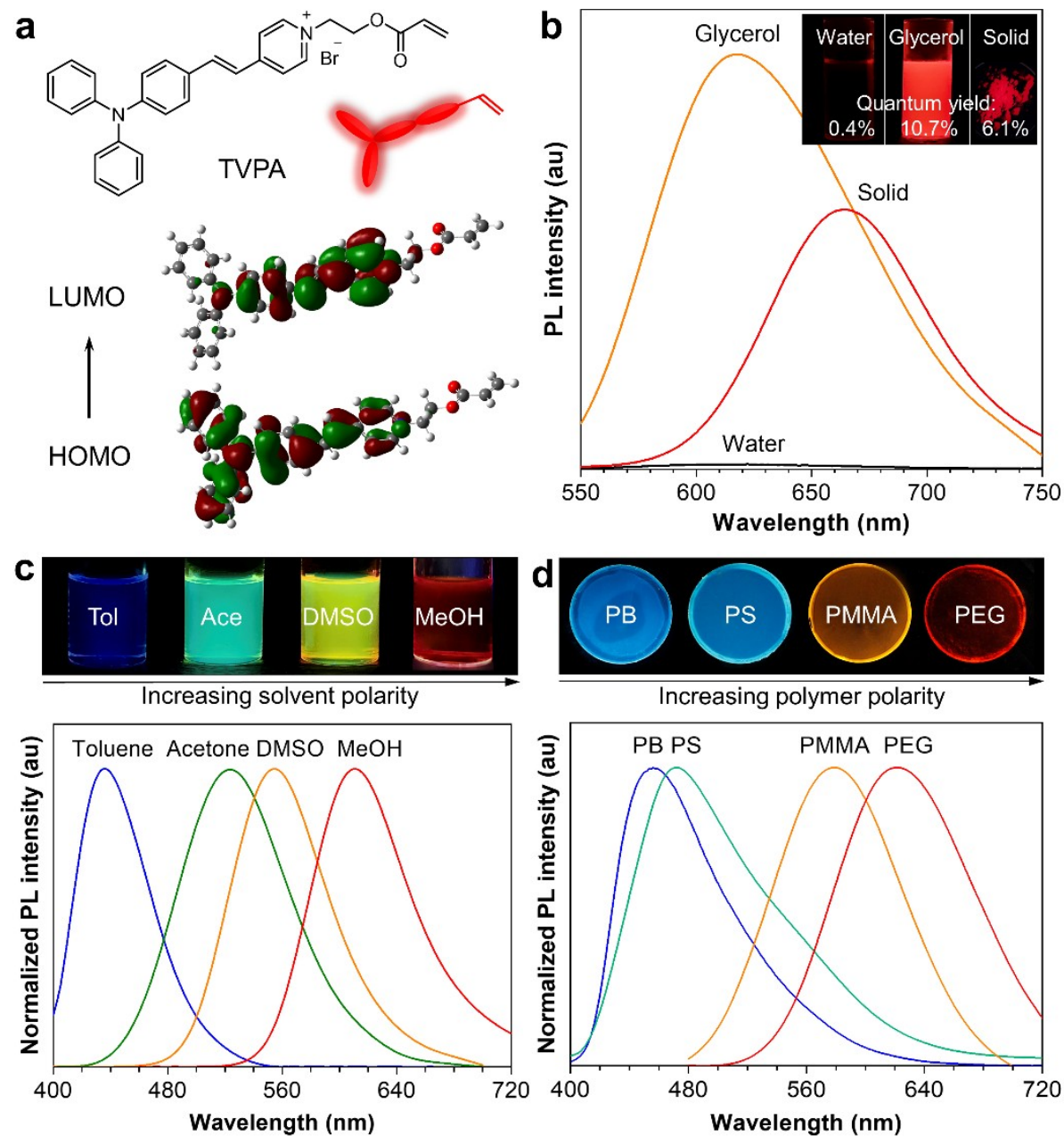

Figure 2. Chemical structure and photophysical response of TVPA probe. a, Chemical structure of TVPA and its theoretical calculated molecular orbital amplitude plots of HOMO and LUMO. b, PL spectra of TVPA in water (10 $\mu \mathrm{M})$, viscous glycerol $(10 \mu \mathrm{M})$ and the solid state. Inset: The corresponding fluorescent photographs and quantum yield (QY) of TVPA. c-d, Fluorescent photographs and photoluminescence (PL) spectra of TVPA (c) in solvents with different polarities: Toluene (Tol), acetone (Ace), dimethyl sulfoxide (DMSO) and methanol (MeOH) and (d) in spin-coated polymer films with various polarities: polybutadiene (PB), polystyrene (PS), poly(methyl methacrylate) (PMMA) and poly(ethylene glycol) (PEG). 
a

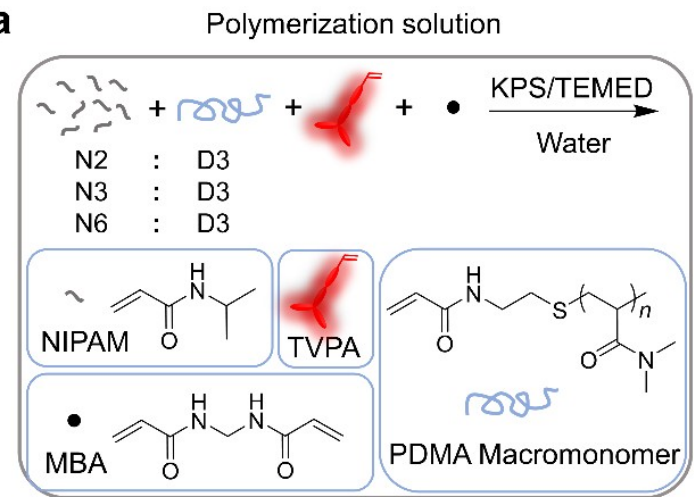

As-prepared hydrogel $\left(G_{a}\right)$

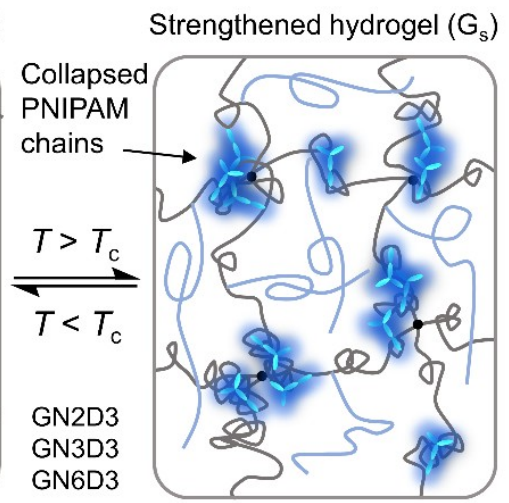

b
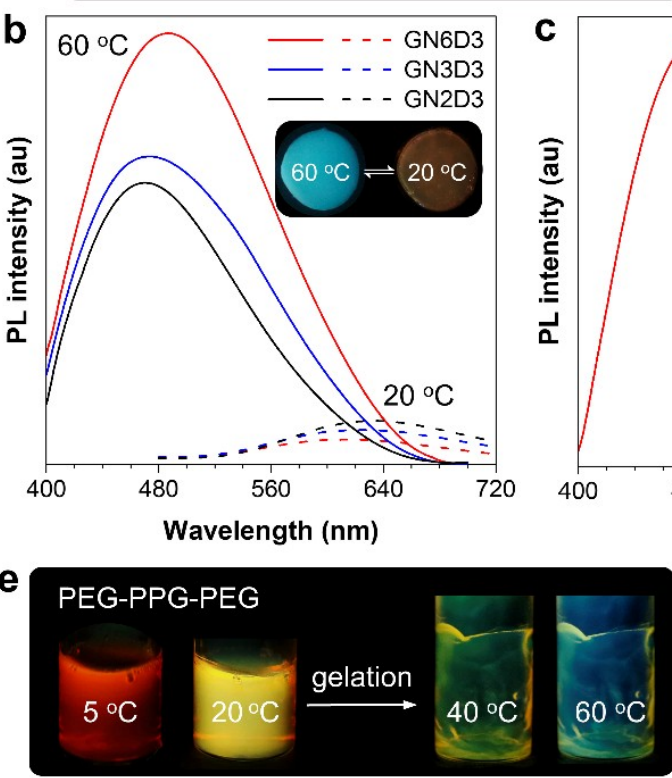

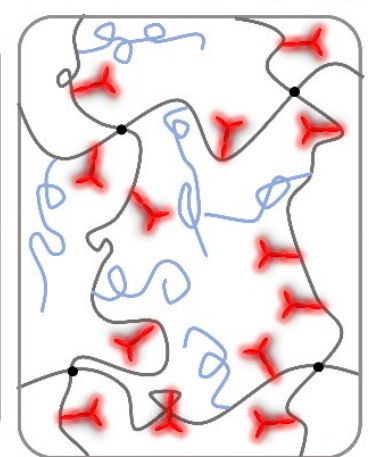

d
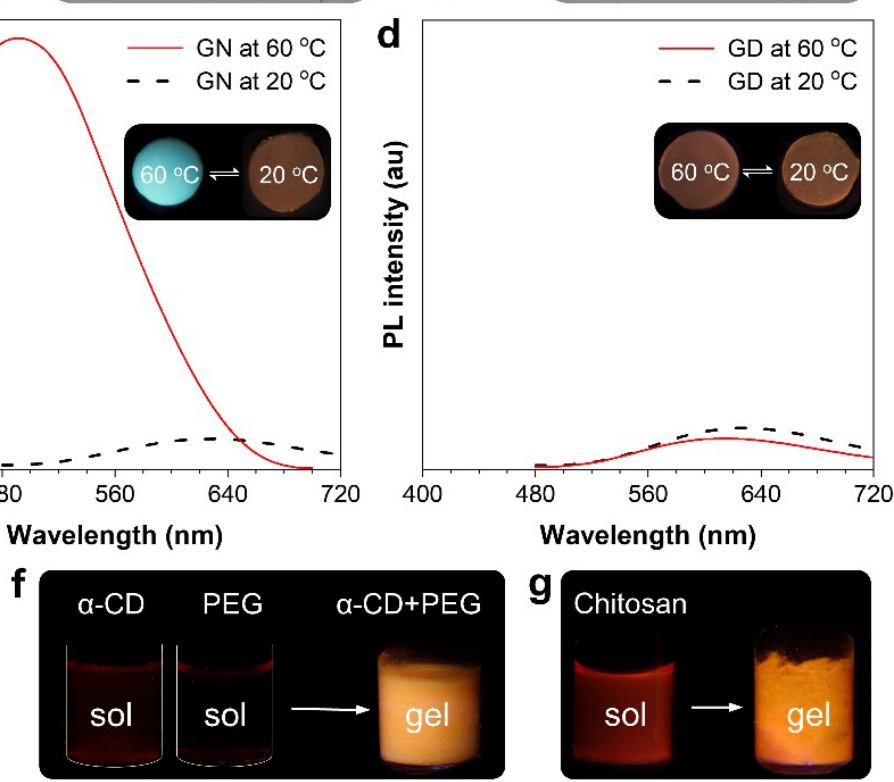

th $(\mathrm{nm})$

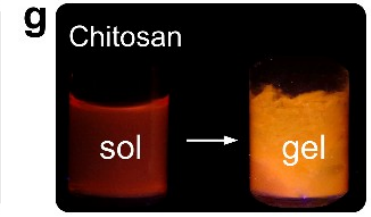

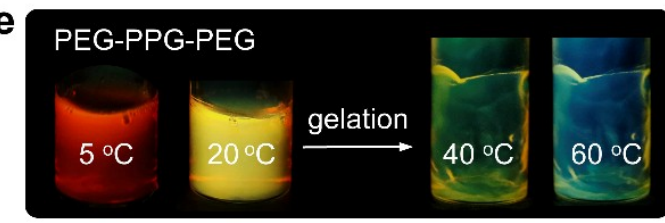

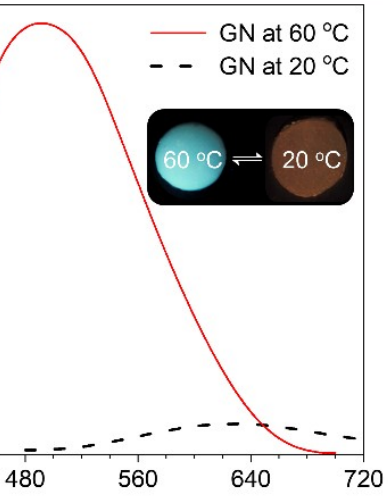

Figure 3. Schematic illustration of design principle and visualization of hydrophilicity-hydrophobicity transformation in aqueous medium. a, TVPA was incorporated into the crosslinked NIPAM polymer network by free radical polymerization of NIPAM, TVPA, PDMA macromonomer and MBA crosslinker. The collapse of the resulting PNIPAM network is induced above its transition temperature $\left(T_{\mathrm{c}}\right)$ and the emission of the incorporated TVPA is expected to be enhanced and blue-shifted in the crowded and hydrophobic PNIPAM domains. Three hydrogels were prepared with different weight fractions of NIPAM and PDMA. b-d, PL spectra of TVPAincorporated (b) GN2D3, GN3D3 and GN6D3, (c) GD and (d) GN hydrogels at $20{ }^{\circ} \mathrm{C}$ and $60{ }^{\circ} \mathrm{C}$ in water $\left(\lambda_{\text {exc }}=\right.$ $380 \mathrm{~nm}$ ). Inset: fluorescent photos of hydrogels at $20^{\circ} \mathrm{C}$ and $60{ }^{\circ} \mathrm{C}$ taken under $365 \mathrm{~nm} \mathrm{UV}$ light. e-f, Fluorescent photos of various hydrogels with stimuli-responsive gelation. e, PEG-PPG-PEG solution is gelled at $40{ }^{\circ} \mathrm{C}$ and its thermoresponsive emission color is gradually blue-shifted with increasing temperature. f, A mixture of PEG and $\alpha$ $\mathrm{CD}$ solutions is gelled after the complex formation for $24 \mathrm{~h} . \mathbf{g}$, Chitosan solution (pH 5.6) is gelled upon the addition of sodium hydroxide solution (0.01 M). 

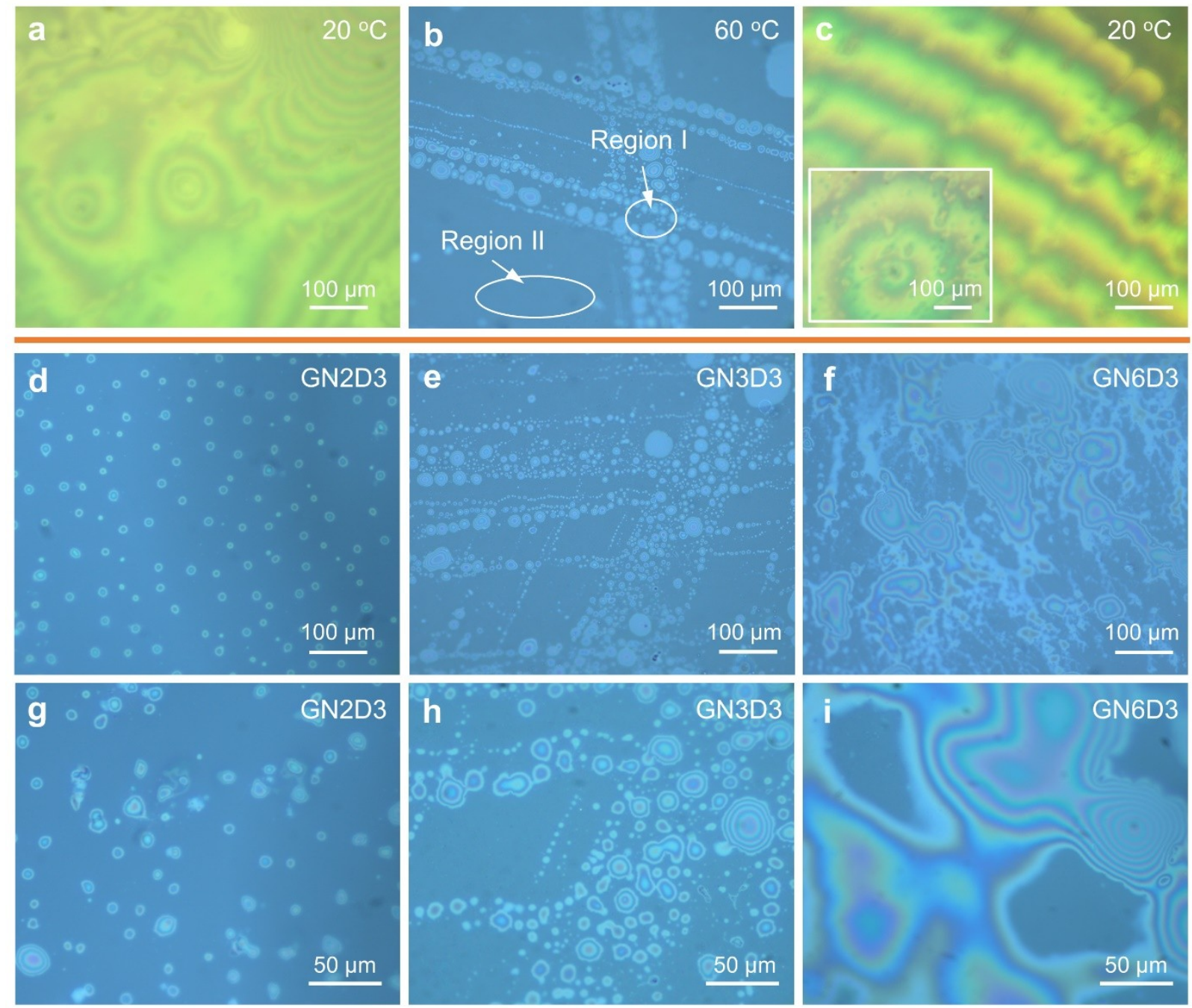

Figure 4. Visualization of microphase separation by fluorescent microscopy. a-c, Fluorescent images of thermoresponsive hydrogels (a) in the preparation state, (b) after immersing in water at $60{ }^{\circ} \mathrm{C}$, and (c) after overnight recovery at room temperature using GN3D3 as a representative example. d-i, Fluorescence images of microphase-separated hydrogels of (d, g) GN2D3, (e,h) GN3D3 and (f,i) GN6D3 after immersing in water at $60{ }^{\circ} \mathrm{C}$ for $30 \mathrm{~min}$. 

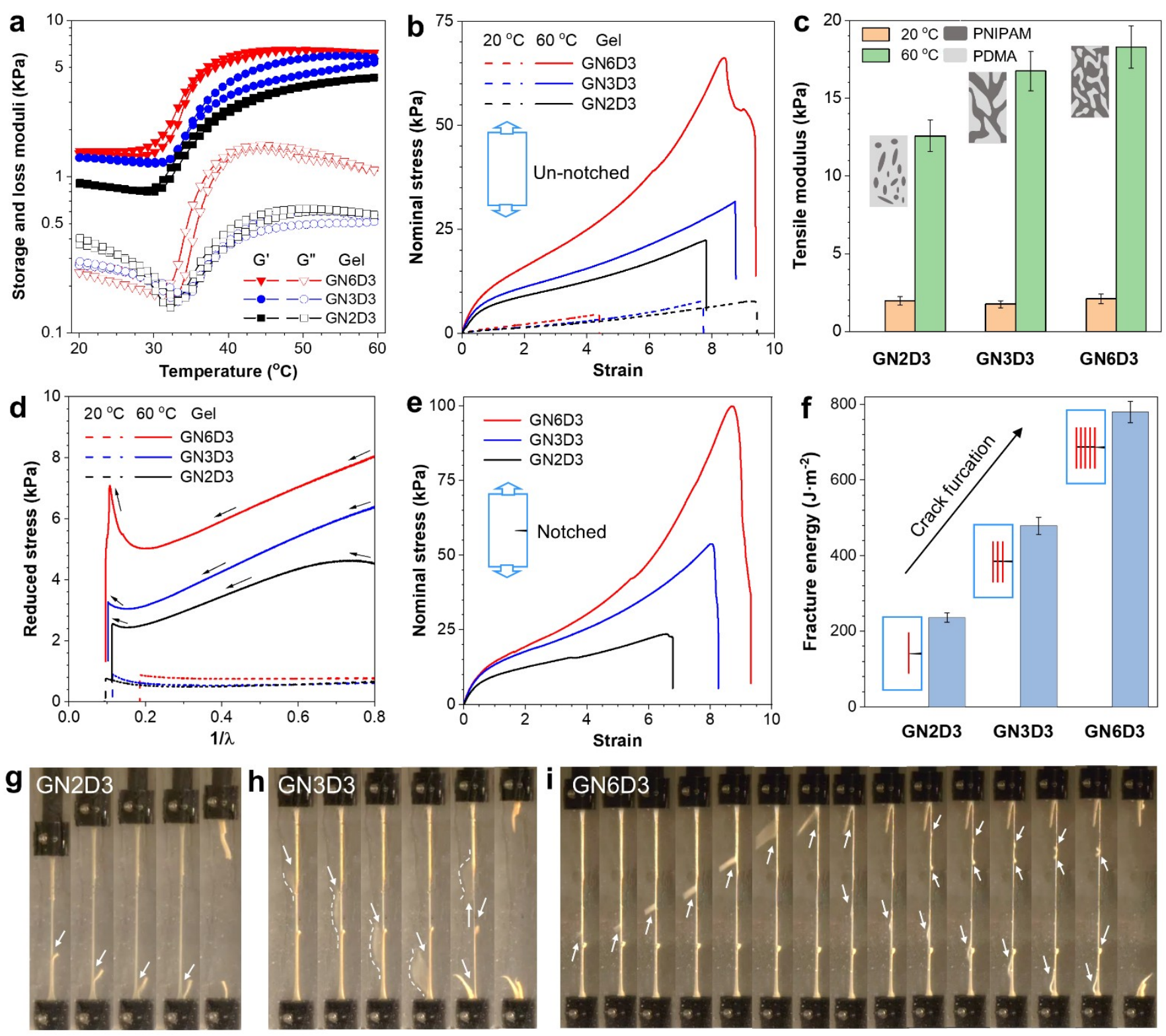

Figure 5. Thermoresponsive viscoelastic, large strain behavior and crack multifurcation. a, Temperaturedependent shear storage (solid symbols, G') and loss (open symbols, G”) moduli of GN2D3 (black), GN3D3 (blue) and GN6D3 (red) with a heating/cooling rate of $0.2^{\circ} \mathrm{C} / \mathrm{min}$. b, Tensile stress-strain curves of GN2D3, GN3D3 and GN6D3 at $20{ }^{\circ} \mathrm{C}$ and $60{ }^{\circ} \mathrm{C}$. c, Tensile moduli of GN2D3, GN3D3 and GN6D3 at $20{ }^{\circ} \mathrm{C}$ (yellow) and $60{ }^{\circ} \mathrm{C}$ (green) Inset: Microphase separation patterns referring to the actual situation. d, Plot of reduced stress versus $1 / \lambda$ at $20{ }^{\circ} \mathrm{C}$ and $60{ }^{\circ} \mathrm{C}$ corresponding to the stress-strain curves in (b). e, Tensile stress-strain curves of GN2D3, GN3D3 and GN6D3 with pre-crack at $60{ }^{\circ} \mathrm{C}$. f, Comparative analysis of fracture energies of pre-cracked hydrogels. Inset: Schematic diagram of crack furcation referring to the actual situation. $\mathbf{g}-\mathbf{i}$, Pictures taken during tensile tests of precracked hydrogels in paraffin oil baths at $60{ }^{\circ} \mathrm{C}$, which record the crack propagation and multifurcation process of (g) GN2D3, (h) GN3D3 and (i) GN6D3. 

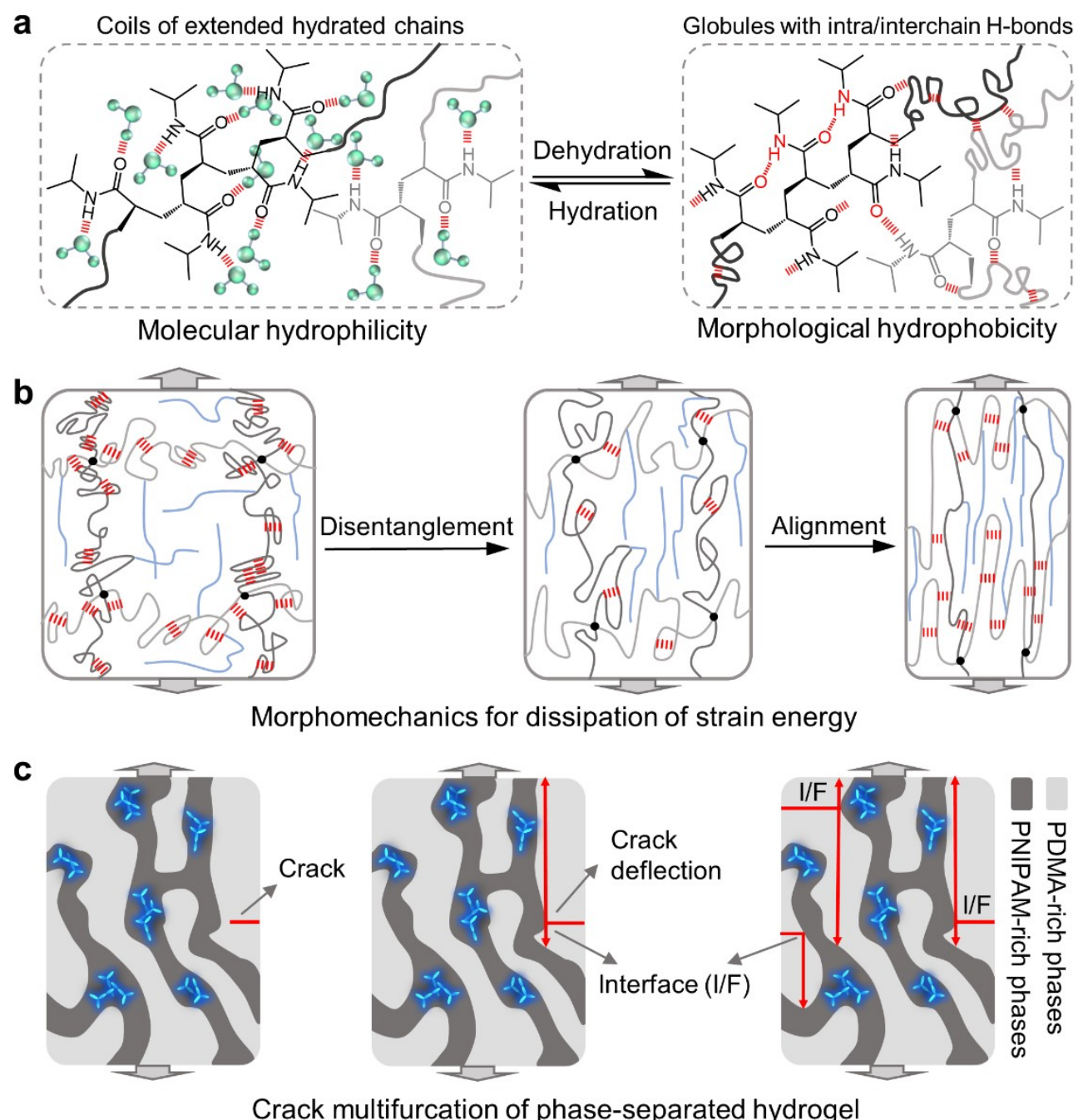

Crack multifurcation of phase-separated hydrogel

Figure 6. Proposed mechanism for thermoresponsive toughening and crack multifurcation. a, The reversible transformation of molecular hydrophilicity and morphological hydrophobicity during thermally induced hydration and dehydration of PNIPAM network. The previous hydrogen bond accepters in PNIPAM for water molecules are occupied by intra and interchain hydrogen bonds above the LCST. b, Disruption of noncovalent interactions, mainly hydrogen bonds, contributes to the strain energy dissipation during the disentanglement of collapsed PNIPAM network. The regained noncovalent interactions during the orientation and alignment of the highly stretched PNIPAM network also reinforces the tensile strength before fracture. c, Proposed multifurcation mechanism of microphase separated hydrogels induced by pre-crack. Pre-crack propagates until it meets the weak interface of PDMA-rich (light grey) and PNIPAM-rich (dark grey) phases and induces crack deflection along the stretching direction. Increasing fracture surface area by crack multifurcation retards the fracture and enlarges fracture energy. 
The table of contents entry: The direct visualization of microphase separation and easy differentiation of hydrophilicity-hydrophobicity transformation has been achieved in a facile, high-contrast and noninvasive manner by using luminogens with aggregation-induced emission as fluorescent indicators. Based on the morphological observation and mechanical study, the concept of morphomechanics with a comprehensive mechanism clarification is proposed.

Hydrophilicity-Hydrophobicity Transformation, Thermoresponsive Morphomechanics and Crack Multifurcation Revealed by AIEgens in Mechanically Strong Hydrogels

Yubing Hu, Lucile Barbier, Zhao Li, Xiaofan Ji, Heiva Le Blay, Dominique Hourdet, Nicolas Sanson, Jacky W. Y. Lam*, Alba Marcellan*, and Ben Zhong Tang*

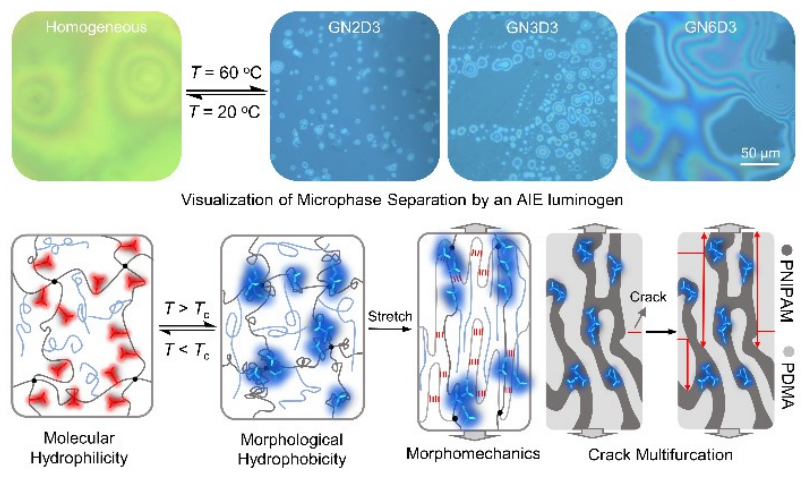

Table of Contents 